\title{
SUPERGRAVITY AND THE INVERTED GAUGE HIERARCHY
}

\author{
Burt A. OVRUT \\ The Institute for Advanced Study, Princeton, NJ 08540, USA \\ Stuart RABY ${ }^{1}$ \\ Randall Laboratory of Physics, University of Michigan, Ann Arbor, MI 48109, USA
}

Received 4 October 1982

Revised manuscript received 5 November 1982

\begin{abstract}
We generalize a class of O'Raifeartaigh models with Witten's inverted gauge hierarchy to $N=1$ supergravity. Radiative corrections induce a grand unification scale that is several orders of magnitude below the Planck mass. This scale is obtained naturally, without having to fine tune the parameters.
\end{abstract}

In globally supersymmetric models of the O'Raifeartaigh type [1] (with mass, coupling and gauge parameters $M, \lambda_{i}$ and $g$ respectively) the vacuum expectation value (VEV) of a singlet field $(X)$ is not determined at the true level. Since supersymmetry is spontaneously broken in these models, this VEV will be determined by radiative corrections. The renormalization group (RG) improved one-loop effective potential has the form [2]

$$
\begin{aligned}
V_{\text {eff }} & =V\left(\lambda_{i}(t), M(t)\right) \mathrm{e}^{4 t} \\
& =V\left(\lambda_{i}, M\right)\left[1+F\left(\lambda_{i}, g\right) t+\mathrm{O}\left(t^{2}\right)\right],
\end{aligned}
$$

where $V$ is the tree level potential and $\lambda_{i}(t), M(t)$ are the running coupling and mass parameters evaluated at scale $t=\ln (X / M)$. For $F\left(\lambda_{i}, g\right)>0$ the effective potential is minimized for $X=0$ [3]. For $F\left(\lambda_{i}, g\right)<0$ the effective potential is a decreasing function for small values of $t$. Since the potential in globally supersymmetric theories must be non-negative, $V_{\text {eff }}$ either (1) asymptotically approaches some non-negative value or (2) goes through a minimum and then increases. For a minimum to occur there must be some $t=t_{0}$ $>0$ for which

\footnotetext{
1 On leave of absence from Los Alamos National Laboratory,
} Los Alamos, NM 87545, USA.

$$
F\left(\lambda_{i}\left(t_{0}\right), g\left(t_{0}\right)\right)=0
$$

Witten [4] has argued that the existence of such a minimum could provide a solution to the gauge hierarchy problem since the ratio $X / M$ (evaluated at the minimum) would naturally be exponentially large. The RG analysis of Witten's model and a demonstration of the existence of $t_{0}$ satisfying eq. (2) has been given by Yamagishi [5].

Recently, this model has been reexamined by Hall and Hinchliffe [6]. Although they agree analytically with the above authors they find that a numerical evaluation of the RG equations leads to the conclusions that (1) the natural (without fine tuning) value of the ratio $X / M$ is of $O(15)$, clearly insufficient for grand unified models and (2) in order for the value of $X / M$ to be of $\mathrm{O}\left(10^{14}\right)$ (or $\mathrm{O}\left(10^{7}\right)$ in geometric hierarchy models) it is necessary to fine tune coupling parameters $\lambda_{i}$ to be very close to a UV repulsive fixed point (they must be in an area that is a fraction $10^{-6}$ of the available perturbative domain). It follows that Witten's mechanism cannot naturally lead to the observed mass hierarchy (at least not for the simple models originally proposed). However, these conclusions apply to globally supersymmetric theories only. In this paper we will show that when O'Raifeartaigh models are coupled to $N=1$ supergravity (that is, made locally supersymmetric) then the appropriate exten- 
sion of Witten's mechanism does lead to a naturally large mass hierarchy (in general several orders of magnitude below the Planck mass).

Consider a class of SU(5) models with two adjoint fields $A, Z$ and a singlet field $X$. The superpotential is

$W=X W_{0}+\operatorname{tr} Z W_{1}$,

where $W_{0}\left(W_{1}\right)$ is a function of $\operatorname{tr} A^{2}\left(A^{2}\right)$ (in Witten's model $W_{0}=\lambda_{2}\left(\operatorname{tr} A^{2}-M^{2}\right)$ and $\left.W_{1}=\lambda_{1} A^{2}\right)$. Coupling to $N=1$ supergravity, the tree level potential energy is given by [7]

$$
\begin{aligned}
V & =\exp \left(K / M_{\mathrm{p}}^{2}\right)\left[\left|W_{0}\right|^{2}\left(1-\frac{|X|^{2}}{M_{\mathrm{p}}^{2}}+\frac{|X|^{4}}{M_{\mathrm{p}}^{4}}\right)+\operatorname{tr}\left|W_{1}\right|^{2}\right. \\
& -\frac{1}{5}\left|\operatorname{tr} W_{1}\right|^{2}-M_{\mathrm{p}}^{-2}\left|\operatorname{tr} W_{1} Z\right|^{2}+M_{\mathrm{p}}^{-4}\left|\operatorname{tr} W_{1} Z\right|^{2} \operatorname{tr}|Z|^{2} \\
& +\left(\frac{|X|^{2}}{M_{\mathrm{p}}^{2}}+M_{\mathrm{p}}^{-2} \operatorname{tr}|Z|^{2}-1\right)\left(\frac{W_{0} X}{M_{\mathrm{p}}^{2}}\left(\operatorname{tr} Z W_{1}\right)^{\dagger} \text { h.c. }\right) \\
& \left.+\frac{|X|^{2}\left|W_{0}\right|^{2}}{M_{\mathrm{p}}^{4}} \operatorname{tr}|Z|^{2}+\frac{|X|^{2}}{M_{\mathrm{p}}^{4}}\left|\operatorname{tr} Z W_{1}\right|^{2}+\operatorname{tr}\left|D_{A} W\right|^{2}\right]
\end{aligned}
$$

Note that expression (4) becomes the usual

O'Raifeartaigh potential energy in the limit that $M_{\mathrm{p}}$ $\rightarrow \infty$. We now examine whether or not there is a direction in field space for which the quadratic (mass) terms in $X$ and $Z$ vanish. We find that these terms vanish if and only if

$W_{0}^{\dagger} Z=X\left(W_{1}^{\dagger}-\frac{1}{5}\left(\operatorname{tr} W_{1}\right)^{\dagger} 1\right)$

and

$D_{A} W=X\left(D_{A} W_{0}\right)+\operatorname{tr} Z\left(D_{A} W_{1}\right)=0$,

where $D_{A} W$ is the Kähler covariant derivative of $W$ with respect to $A$ [7]. Combining eqs. (5) and (6) im. plies that

$W_{0}^{\dagger} D_{A} W_{0}+\operatorname{tr} W_{1}^{\dagger} D_{A} W_{1}=0$,

which is an equation involving field $A$ only. This region of field space minimizes the potential energy in the $A$ direction. If we denote the solution of eq. (7) by $\langle A\rangle$, then the potential energy in the $X, Z$ direction (for which the quadratic terms vanish) is

$$
\begin{aligned}
V= & \exp \left(M_{\mathrm{p}}^{-2} \operatorname{tr}|\langle A\rangle|^{2}\right) \\
& \times\left.\left(\left|W_{0}\right|^{2}+\operatorname{tr}\left|W_{1}\right|^{2}-\frac{1}{5}\left|\operatorname{tr} W_{1}\right|^{2}\right)\right|_{(A)} \\
& \times\left[1+\left(1 / 2 M_{\mathrm{p}}^{4}\right)\left(|X|^{2}+\operatorname{tr}|Z|^{2}\right)^{2}+O\left(M_{\mathrm{p}}^{-6}\right)\right]
\end{aligned}
$$

Note that expression (8) is independent of $X$ and $Z$ in the limit that $M_{\mathrm{p}} \rightarrow \infty$. It follows that the region of field space defined by eqs. (5) and (6) is the supergravity generalization of the "flat direction" in the globally supersymmetric $O$ 'Raifeartaigh model. We emphasize that the sign of the $M_{\mathrm{p}}^{-4}$ term in (8) is positive and that the tree level VEVs for $X$ and $Z$ vanish. We now calculate $\operatorname{VEV}\langle A\rangle$ from eq. (7). For concreteness, we henceforth assume

$$
W_{0}=\lambda_{2}\left(\operatorname{tr} A^{2}-M^{2}\right), \quad W_{1}=\lambda_{1} A^{2} .
$$

We find that

$$
\begin{gathered}
\langle A\rangle=\left[1-\left(\frac{\lambda_{1}^{2}}{\lambda_{1}^{2}+30 \lambda_{2}^{2}}\right) \frac{M^{2}}{4 M_{\mathrm{p}}^{2}}+\mathrm{O}\left(M_{\mathrm{p}}^{-4}\right)\right] \\
\times \frac{\lambda_{2} M}{\left(\lambda_{1}^{2}+30 \lambda_{2}^{2}\right)^{1 / 2}}\left(\begin{array}{ccccc}
2 & & & \\
& 2 & & & \\
& & & & \\
& & & & \\
& & & & -3
\end{array}\right)
\end{gathered}
$$

In the limit that $M_{\mathrm{p}} \rightarrow \infty$ expression (10) becomes the usual tree level VEV in the O'Raifeartaigh model (see, for example, ref. [8]) which breaks $S U(5)$ down to $\mathrm{SU}(3) \times \mathrm{SU}(2) \times \mathrm{U}(1)$. Note that the $\mathrm{O}\left(M_{\mathrm{p}}^{-2}\right)$ correction is proportional to the $O(1) \mathrm{VEV}$ and, hence, further breakdown of the $\mathrm{SU}(3) \times \mathrm{SU}(2) \times \mathrm{U}(1)$ symmetry due to gravitation does not occur. The VEV for $Z$ can now be evaluated using eqs. (5) and (10). We find

$$
\begin{aligned}
\langle Z\rangle & =\left(1+\frac{M^{2}}{2 M_{\mathrm{p}}^{2}}+O\left(M_{\mathrm{p}}^{-4}\right)\right) \frac{\lambda_{2}}{\lambda_{1}}\langle X\rangle \\
& \times\left(\begin{array}{lllll}
2 & & & \\
& 2 & & & \\
& & 2 & & \\
& & & -3 & \\
& & & -3
\end{array}\right) .
\end{aligned}
$$

In the limit that $M_{\mathrm{p}} \rightarrow \infty$ expression (11) becomes the usual tree level VEV in the O'Raifeartaigh model which breaks $S U(5)$ down to $S U(3) \times S U(2) \times U(1)$. Once again, no further breakdown of symmetry due to gravi- 
tational corrections occurs.

We now want to include the one-loop radiative corrections to (8). The $O(1)$ corrections have been calculated by Witten [4]. Since supergravity is non-renormalizable it is not clear what meaning (if any) radiative corrections to the effective potential involving vertices of $\mathrm{O}\left(M_{\mathrm{p}}^{-1}\right)$ have. In this paper we simply ignore such corrections, an approximation that is presumably valid as long as $|X| \lesssim M_{\mathrm{p}}$. We henceforth work in this approximation. The one-loop effective potential is then given by

$$
\begin{aligned}
& V_{\text {eff }}=\exp \left(M_{\mathrm{p}}^{-2} \operatorname{tr}|\langle A\rangle|^{2}\right)\left(\frac{\lambda_{1}^{2} \lambda_{2}^{2} M^{4}}{\lambda_{1}^{2}+30 \lambda_{2}^{2}}+O\left(M_{\mathrm{p}}^{-2}\right)\right) \\
& \quad \times\left\{\left[1+\frac{3}{4 \pi^{2}} \frac{\lambda_{2}^{2}}{\lambda_{1}^{2}+30 \lambda_{2}^{2}}\left(29 \lambda_{1}^{2}-50 g^{2}\right) \ln \left(\frac{|X|}{M}\right)\right]\right. \\
& \left.\quad+\frac{1}{2 M_{\mathrm{p}}^{4}}\left(\frac{\lambda_{1}^{2}+30 \lambda_{2}^{2}}{\lambda_{1}^{2}}\right)^{2}|X|^{4}+O\left(M_{\mathrm{p}}^{-6}\right)\right\}
\end{aligned}
$$

This expression is only valid for fields $|X| \ll M_{\mathrm{p}}$. We can extend the range of validity of (12) using the renormalization group. The renormalization group improved one-loop effective potential is given by

$$
\begin{gathered}
V_{\mathrm{eff}}=\exp \left(M_{\mathrm{p}}^{-2} \operatorname{tr}|\langle A\rangle|^{2}\right)\left[\left(\frac{\lambda_{1}(t)^{2} \lambda_{2}(t)^{2} M(t)^{4}}{\lambda_{1}(t)^{2}+30 \lambda_{2}(t)^{2}}\right) \mathrm{e}^{4 t}\right. \\
\left.+\frac{M^{4}}{2 M_{\mathrm{p}}^{4}} \frac{\lambda_{2}^{2}}{\lambda_{1}^{2}}\left(\lambda_{1}^{2}+30 \lambda_{2}^{2}\right)|X|^{4}+\mathrm{O}\left(M_{\mathrm{p}}^{-6}\right)\right], \quad
\end{gathered}
$$

where $\lambda_{i}(t), M(t)$ are running parameters, $t=\ln (|X| / M)$ and we have dropped an irrelevant constant of $\mathrm{O}\left(M_{\mathrm{p}}^{-2}\right)$. Note that the parameters $\lambda_{i}$ associated with $O\left(M_{\mathrm{p}}^{-4}\right)$ do not run, in accordance with the approximation discussed above. Expression (13) is, in general, valid for fields $|X| \lesssim M_{\mathrm{p}}$. This is sufficient for our needs. Differentiating $V_{\text {eff }}$ with respect to $t$ we find that, to $\mathrm{O}\left(M_{\mathrm{p}}^{-4}\right), \operatorname{VEV}\langle X\rangle$ is the solution of equation

$$
\begin{aligned}
& \left(\frac{\lambda_{1}\left(t_{0}\right)^{2} \lambda_{2}\left(t_{0}\right)^{2} M\left(t_{0}\right)^{4}}{\lambda_{1}\left(t_{0}\right)^{2}+30 \lambda_{2}\left(t_{0}\right)^{2}} \mathrm{e}^{4 t_{0}}\right) F\left(\lambda_{i}\left(t_{0}\right), g\left(t_{0}\right)\right) \\
& =-\frac{2 M^{4}}{M_{\mathrm{p}}^{4}} \frac{\lambda_{2}^{2}}{\lambda_{1}^{2}}\left(\lambda_{1}^{2}+30 \lambda_{2}^{2}\right)\langle X\rangle^{4},
\end{aligned}
$$

where

$$
\begin{aligned}
& F\left(\lambda_{i}(t), g(t)\right)=\frac{3}{4 \pi^{2}} \frac{\lambda_{2}(t)^{2}}{\lambda_{1}(t)^{2}+30 \lambda_{2}(t)^{2}} \\
& \quad \times\left[29 \lambda_{1}(t)^{2}-50 g(t)^{2}\right] .
\end{aligned}
$$

In the limit $M_{\mathrm{p}} \rightarrow \infty$ eq. (14) becomes identical to (2).

To solve eq. (14) we must now investigate the RG equations for $\lambda_{i}$ and $g$. Let $\alpha=g^{2} / 4 \pi$ and $\alpha_{i}=\lambda_{i}^{2} / 4 \pi$. Using variables $u_{i}=\alpha_{i} / \alpha$ and

$s=\frac{1}{5} \ln [1+(5 / 2 \pi) \alpha t]$

gives [5]

$$
\begin{aligned}
& \mathrm{d} \alpha(t) / \mathrm{d} t=-(5 / 2 \pi) \alpha(t)^{2}, \\
& \mathrm{~d} u_{1}(s) / \mathrm{d} s=u_{1}(s)\left[21 u_{1}(s)+8 u_{2}(s)-25\right], \\
& \mathrm{d} u_{2}(s) / \mathrm{d} s=u_{2}(s)\left[\frac{84}{5} u_{1}(s)+56 u_{2}(s)-15\right] .
\end{aligned}
$$

The phase diagram for eqs. (18) and (19) is shown in fig. 1 . We are interested in theories for which $F\left(\lambda_{i}, g\right)$ $<0$. It follows that we must restrict our starting parameters to be to the left of the $F=0$ line. Region I has two kinds of RG trajectories, (a) those that intersect the $F=0$ line within the perturbative domain $(0$ $\leqslant u_{i} \lesssim 2$ or 3 ) and (b) those that do not. In region II all RG trajectories are attracted to the origin. Let us first consider the limit that $M_{\mathrm{p}} \rightarrow \infty$. In this case initial parameters chosen in region II lead to a run-away solution for $\langle X\rangle$. For a stable mass hierarchy to develop

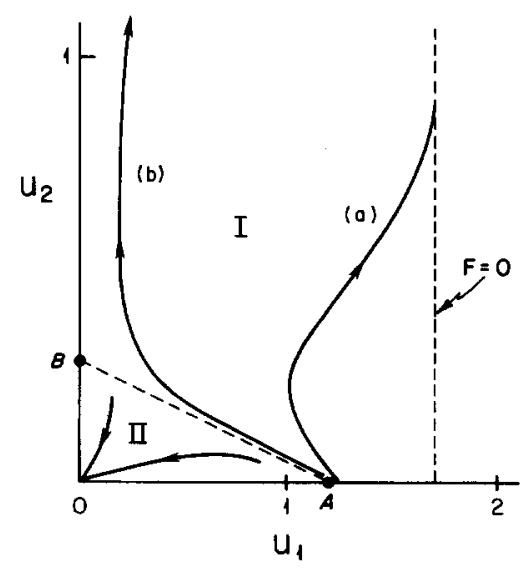

Fig. 1. Renormalization group trajectories in the $u_{1}, u_{2}$ plane. Points $O((0,0))$ and $A((25 / 21,0))$ are ultraviolet attractive and repulsive fixed points respectively. Point $\mathrm{B}((0,15 / 56))$ is a saddle point. The $F=0$ line occurs at $u_{1}=50 / 29$. 
it is necessary to take initial parameters in region I. Hall and Hinchliffe [6] have shown that for trajectories of type (a) to intersect the $F=0$ line after evolving for a sufficiently long time to account for the observed mass hierarchy ( $t_{0} \approx 33$ in Witten's model) it is necessary to fine tune the initial parameters to be very close to UV repulsive fixed point $A$. All other initial parameters lie on trajectories that intersect the $F=0$ line for values of $t_{0} \ll 33$ or do not intersect it at all (at least within the perturbative domain). Thus, for $M_{\mathrm{p}} \rightarrow \infty$ (no supergravity), Witten's mechanism does not lead to a natural solution to the gauge hierarchy problem. Now let $M_{\mathrm{p}}$ be finite (turn on supergravity). If our initial parameters lie in region $I$ it is clear that the $\mathrm{O}\left(\mathrm{Mp}_{\mathrm{p}}^{-4}\right)$ term in eq. (14) does not get us out of the above problem (it makes matters worse). Let us re-examine the case when the initial parameters lie in region II. We henceforth assume that $\alpha=1 / 10$. Eq. (17) is easily solved to give

$\alpha(t)=1 / 10(1+t / 4 \pi)$.

For $0 \leqslant u_{1}, u_{2} \leqslant 10^{-1}$ (an area of $\mathrm{O}\left(10^{-1}\right.$ ) of region II and $O\left(10^{-2}-10^{-3}\right)$ of the entire perturbative domain) the quadratic terms in eqs. (18) and (19) can be ignored. In this regime

$u_{1}(t) \approx u_{1}(1+t / 4 \pi)^{-5}$,

$u_{2}(t) \approx u_{2}(1+t / 4 \pi)^{-3}$.

Rewriting expression (15) in terms of $u_{i}(t)$ and $\alpha(t)$ we find, using (20) and (21), that

$F\left(\lambda_{i}(t), g(t)\right) \approx-(1 / 2 \pi)(1+t / 4 \pi)^{-1}$,

as long as $u_{1} / u_{2} \lesssim \mathrm{O}(1)$ (for simplicity, we henceforth assume this to be the case). Similarly, using the solution for $M(t)$ given in ref. [5], we find

$\frac{\lambda_{1}(t)^{2} \lambda_{2}(t)^{2} M(t)^{4}}{\lambda_{1}(t)^{2}+30 \lambda_{2}(t)^{2}} \mathrm{e}^{4 t} \approx(\pi / 75) M^{4} u_{1}(1+t / 4 \pi)^{-2}$.

Eq. (14) then becomes

$\langle X\rangle^{4}=\frac{10^{-4}}{3.6 \pi}\left(\frac{u_{1}}{u_{2}}\right)^{2}\left(1+t_{0} / 4 \pi\right)^{-3} M_{\mathrm{p}}^{4}$.

For $M=10^{3} \mathrm{GeV}$ the solution of eq. (24) is

$$
\begin{aligned}
t_{0} & \approx 33.9+\frac{1}{2} \ln \left(u_{1} / u_{2}\right) \\
& -\frac{3}{4} \ln \left\{1+(1 / 4 \pi)\left[33.9+\frac{1}{2} \ln \left(u_{1} / u_{2}\right)\right]\right\} .
\end{aligned}
$$

Assuming $u_{1} / u_{2}=1$ implies $t_{0} \approx 32.9$ from which it follows that $\langle X\rangle / M$ is of $\mathrm{O}\left(10^{14}\right)$. This is precisely the correct mass hierarchy. (For $M=10^{12}$ in geometric hierarchy models a similar analysis leads to $\langle X\rangle / M$ of $\mathrm{O}\left(10^{6}\right)$, an acceptable result.) Note that since $\langle X\rangle$ is of $\mathrm{O}\left(10^{-2} M_{\mathrm{p}}\right)\left(\mathrm{O}\left(10^{-1} M_{\mathrm{p}}\right)\right.$ in geometric hierarchy models) it lies in the region of field space where we expect all of the above approximations to be valid. For $u_{1} / u_{2} \neq 1$, a wide range of values of this ratio still leads to a reasonable mass hierarchy. For example, when $M=10^{3} \mathrm{GeV}$ then $u_{1} / u_{2}=10^{-3}$ implies that $t_{0} \approx 29.5$. It follows that $\langle X\rangle / M$ is of $\mathrm{O}\left(10^{13}\right)$ which is sufficiently large. Finally, we point out that the restrictions that $0 \leqslant u_{1}, u_{2} \leqslant 10^{-1}$ and $u_{1} / u_{2}$ $\lesssim \mathrm{O}(1)$ were imposed only to simplify the calculations. More generally, the condition that $u_{1}$ and $u_{2}$ both lie in region II is sufficient (subject to some constraints) to assure that a mass hierarchy of approximately the correct size will be achieved.

The reason for this result is clear. For initial parameters in region II the $O(1)$ value of $\mathrm{d} V_{\text {eff }} / \mathrm{d} t$ is everywhere negative. It follows that the $O(1)$ part of $V_{\text {eff }}$ is a slowly decreasing function of $t$. However, the $\mathrm{O}\left(M_{\mathrm{p}}^{-4}\right)$ part of $V_{\text {eff }}$ is an increasing function of $t$. The above calculation shows that this function increases sufficiently quickly to turn over the potential energy and create a minimum. One might expect $\langle X\rangle$ to be of $\mathrm{O}\left(M_{\mathrm{p}}\right)$ but for technical reasons this VEV is naturally smaller than $M_{\mathrm{p}}$ by several orders of magnitude. This is significant since $\langle X\rangle$ now lies in a region of field space where our approximations [(1) ignoring $\mathrm{O}\left(M_{\mathrm{p}}^{-6}\right)$ tree level terms and (2) ignoring radiative corrections involving vertices of $\left.\mathrm{O}\left(M_{\mathrm{p}}^{-1}\right)\right]$ are expected to be valid. Note that region II is precisely the regime where one would normally choose initial parameters (in contrast, initial parameters in region I are, in general, on the edge of the region where perturbation theory is valid). We conclude that when O'Raifeartaigh models are coupled to $N=1$ supergravity then the appropriate extension of Witten's mechanism leads to a naturally large gauge hierarchy of the correct order of magnitude.

Finally we point out that although $\left\langle D_{A} W\right\rangle=0$, both $\left\langle D_{X} W\right\rangle$ and $\left\langle D_{Z} W\right\rangle$ are non-vanishing and proportional to $M^{2}$ (despite the fact that $\operatorname{VEV}\langle X\rangle$ is near the Planck mass). It follows that supersymmetry is spontaneously broken with the scale of this breaking set by $M^{2}$. We conclude that even though $N=1$ super- 
gravity changes $V_{\text {eff }}$ enough to significantly effect the gauge hierarchy, it does not change the occurrence or scale of supersymmetry breaking. These results open the door to the construction of phenomenologically acceptable, locally supersymmetric grand unified theories. In these theories two scales, the supersymmetry breaking scale and the Planck mass, are put in by hand and the grand unified and electro-weak scales generated radiatively. Such models are presently under consideration.

We would like to thank L. Hall for discussing his work with us and for many useful conversations. We also acknowledge the hospitality of the Aspen Center for Physics where this work was carried out. This work was supported in part by the Department of Energy under grant number DE-AC02-76ER02220 and DEAC02-76ER01112.

\section{References}

[1] L. O'Raifeartaigh, Nucl. Phys. B96 (1975) 331.

[2] S. Coleman and E. Weinberg, Phys. Rev. D7 (1973) 1888.

[3] M. Huq, Phys. Rev.D14 (1976) 3548.

[4] E. Witten, Phys. Lett. 105B (1981) 267.

[5] H. Yamagishi, Princeton University preprint (1982); also see M. Einhorn and D.R.T. Jones, University of Michigan preprint (1982);

T. Banks and V. Kaplunovsky, Tel Aviv University preprint (1982).

[6] L. Hall and I. Hinchliffe, L.B.L. preprint (1982).

[7] J. Bagger and E. Witten, Princeton University preprint (1982);

E. Cremmer, S. Ferrara, L. Girardello and A. Van Proeyen, CERN preprint (1982).

[8] S. Dimopoulos and S. Raby, Los Alamos preprint (1982). 